\title{
Analytical Study for Development of Fuel Adulteration Detection System
}

Prof. Ankita H. Harkare

Department of Electronics and Communication Engg, Shri Ramdeobaba College of Engineering and

Management, Nagpur, India

Email: harkareah@rknec.edu

Received: $20^{\text {th }}$ September 2018, Accepted: $11^{\text {th }}$ October 2018, Published: $31^{\text {st }}$ October 2018

\begin{abstract}
Adulteration in various consumable items and fuel has subsequently increased health hazards and illegal monetary gains associated with it. A real time solution with vendors and consumers has therefore become the need of the hour. The probable carcinogenic, teratogenic, and mutagenic effects of adulteration as well as their wide existence in consumables; rapid, high-throughput and portable methods for sensitive detection are immediately required. Microfluidics is an emerging field which can detect adulteration in food and fuel taking microscopic sample and resulting in high resolution result. Microfluidic lab on chip solutions will offer the consumers highly sensitive detection of hazardous adulteration in any commodity.
\end{abstract}

\section{Keywords}

Adulteration, Microfluidics, Microscopic, Lab on Chip

\section{Introduction}

Adulteration is illegitimate or unlicensed introduction of an inferior external material into a superior quality commodity, due to which the commodity fails to hold its original constitution and properties. These external materials or adulterants added thereby degrade the quality of the product. Major fluid adulteration cases have been witnessed in past few years which cause several health hazards as well as economical hazards. Milk, edible oil, fuel etc. are some of the fluids which are adulterated on a regular basis to generate easy and fast monetary gains. Similarly, food items are prone to mycotoxin adulteration, bestowing health hazards[1]. Though Fuel adulteration does not pose direct impact on human health, it affects human health indirectly through pollution. Also, adulterated fuel is a threat on engine life of the vehicle. Hence, it can be concluded that adulteration in any form causes direct or indirect effect on human health and economy.

Mycotoxins, the secondary metabolites of fungi and the major fungal genera producing them include Aspergillus spp., Fusarium spp. and Penicillium spp. Numerous mycotoxins are produced by these molds including aflatoxins (AFs), deoxynivalenol (DON), zearalenone (ZEA), fumonisin B1 (FB1), ochratoxin A (OTA) and citrinin (CIT), nearly all of these are venomous for humans [2]. Conservative methods for the identifying toxins in agricultural products are techniques that are chromatographic-based, including thin-layer chromatography (TLC), high performance liquid chromatography (HPLC), gas chromatography coupled with mass spectrometry (GCeMS) [3]. Due to high time consumption and extensive sample preparation procedures these conventional methods are not suitable for long run. Moreover, detection requires trained personnel to operate such set- ups. The ease of operation and small sample sizes has made microfluidics the choice that needs to be explored. The adulterated food item needs to be converted into fluid form to determine adulteration through microfluidics.

A report published by Delhi-based Centre for Science and Environment (CSE) in 2014 claims that Fuel Adulteration is widespread in India and is an eye opener [4]. The problem is compounded by the unresponsiveness approach of government agencies and authorities as well as related the oil companies. The 'official' failure rate in samples of fuel due to adulteration in NCR region around Delhi -- Faridabad, Gurgaon and beyond -- is found to be as high as $26 \%$. If a fuel tanker with a capacity of 12,000 liters is contaminated with only 5 per cent kerosene, the profit would be still as attractive as Rs 7,200 per tanker at the current level of prices. Similarly, adulteration of petrol with 15 per cent low aromatic naphtha can earn Rs 25,000 per day.

According to executive director of the country's top oil policy body, the Oil Coordination Committee (OCC), adulteration of petroleum products lead around 40,000 crores per year scam in India. A startling disclosure in 2015 revealed Rs. 50,000crore loss to subsidies which was caused by diversion of 40-45 percent of pds kerosene for adulteration. Crude Oil is the basic source to derive conventional fuels; where several fractions of it are produced by continuous distillation: Petrol (gasoline), kerosene (kerosene, paraffin oil), gas oil (heavy oil), vacuum gas oil, naphtha, lubricating oil and residue. These fractions are further processed through conversion, reforming and treatment processes to increase the yields and adjust the chemical composition of transport fuels and to remove the trace impurities [5]. It was found that kerosene is the major adulterant in diesel fuel as it blends perfectly and hence not easily detected [6]. However, it was found that the dynamic viscosity of pure 
diesel is less as compared to diesel adulterated with kerosene. Thus, dynamic viscosity can be one of the parameter in determining adulteration in diesel fuel.

Researchers have explored numerous methods to detect adulteration in fuel such as density measurement method, fiber grating sensor technology, emission testing, filter paper method, gas chromatography, ash contamination determination etc. [7] [8] [9] Since the above methods are usually lab based; they tend to be bulkier solutions as well as increase the cost of the system and hence cannot be used in real time. Considering the above scenario, microfluidics can be selected in determining the adulteration as well as providing a highly sensitive and robust real time solution.

\section{Physics Behind Microfluidics}

Microfluidics is a multidisciplinary field which includes fluid mechanics, thermodynamics, electrostatics, chemistry, as well as material science [10]. In the recent years, lab on chip applications have taken into consideration the flow of micofluid in microstuctures and nanomaterials required to fabricate these structures [11,12]. Microfluidics involves analysis of certain dimensionless numbers which can determine the flow dynamics of the fluid in mirochannel [13]. These important dimensionless numbers are:

1. Reynolds number [14]

2. Peclet number [15]

3. Capillary number [16]

4. Weber number [17]

5. Cetane Number in case of fuel

The laminer flow of fluid is an important parameter in microchannels and can be described by Navier-Stokes equations [18]. In these type of laminer flow, a parameter which can easily be calculated and depends upon length and width of microchannel needs to be calculated. Dynamic viscoscity is one such parameter which depends upon the width of the microchannel considering heat dissipation as negligible $[19,20]$. Thus,the basis of determining the laminer flow is the Hagen Poiseuille flow equation which considers all the other constants in the equation as constant except the width of the channel which would vary depending upon the two fluids present inside the channel [21].

Where,

$$
\text { Viscosity }=\mathbf{w}^{2} \Delta \mathbf{P} / 2 \mathbf{v}_{\mathrm{m}} \mathbf{L}
$$

$\mathrm{w}$ is the channel width in $\mathrm{mm}$

$\Delta \mathrm{P}$ is the pressure difference between the inlet and outlet in Pascals,

$\mathrm{V}_{\mathrm{m}}$ is the fluid velocity inside the microchannel and

$\mathrm{L}$ is the channel length

Thus, viscosity is directly proportional to the square of the width of the microchannel.

\section{Microfluidic Device: Major Principles and Design}

The device is a Y-shaped channel controlled mixing through diffusion. The device was designed to have two input channels and a common channel. To prevent uncontrolled mixing in the common channel, the laminer flow should be maintained from stream A and B. The design has taken care of this phenomenon [22]. This transport of the specimen between streams A and B should take place only by diffusion so as to keep the speciment with low diffusion coefficients in their respective streams [23]. The device suggested by Goel et.al. [24], has width of the input channels was $25 \mu \mathrm{m}$ and the common channel $50 \mu \mathrm{m}$ wide and $200 \mu \mathrm{m}$ long. The Reynolds number [14], which is important for characterizing the flow, is given by:

where

$$
\operatorname{Re}=\rho \mathrm{UL} / \mu
$$

$\rho$ is the density of fluid (assuming water as the reference with the value of $1000 \mathrm{~kg} / \mathrm{m} 3$ )

$\mathrm{U}$ is a characteristic flow velocity

$\mathrm{L}$ is a characteristic dimension of the device (in $\mathrm{mm}$ )

The device dimensions are as shown in Figure.1 (b). Figure. 1.a. shows simulation of the device using COMSOL Multiphysics. [24] 


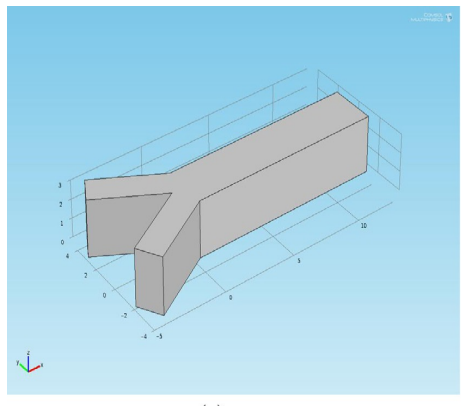

(a)

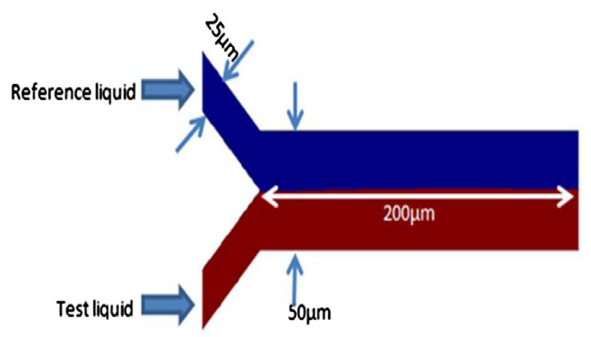

(b)

Figure 1. a: Y-Shaped Channel as Simulated in COMSOL Multiphysics b: Y-Shaped Channel with Actual Dimensions

The fabrication of microfluidic devices is done using glass, silicon, polymers and paper based material [25]. The feasibility of polymer-based materials enables the production of more sophisticated design of microfluidics systems that can be sealed thermally or chemically, compared with silicon and glass [26]. The most widely used polymer is polydimethylsiloxane (PDMS) [27]. The channel shown in Fig1 determines the viscosity of the adulterated fluid by taking a reference fluid of known viscosity into consideration. The reference fluid taken is glycerin. Fluids were infused by syringe pumps [28] and the experimentation setup is as shown in Figure. 2. The syringe pumps can provide constant flow which was set to $60 \mathrm{ml} / \mathrm{h}$ [24]. When two fluids of different viscosities flow through the common channel, higher resistance is exhibited by more viscous fluid than the other fluid. As a result of which, the less viscous fluid will occupy more area in the common channel initially. However, after stabilization, the more viscous fluid will occupy more area with respect to time. Now, the width occupied by both the fluids can be measured and the viscosity of unknown fluid can be determined.

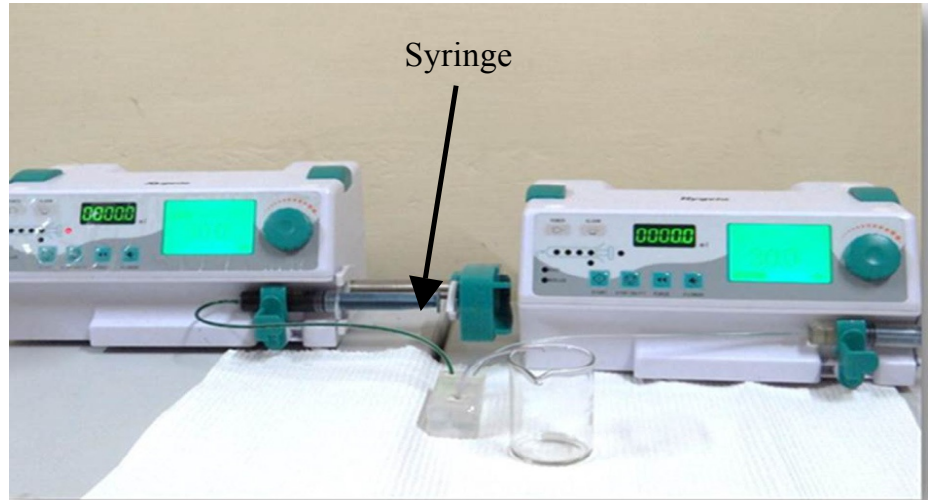

Figure 2: Experimental Set-up to use the Microfluidic Device to Measure the Viscosity of the Given Samples [24]

Figure. 3 shows interface shifts based on the calculated viscosity the amount of adulteration can be predicted.

\section{Glycerin}

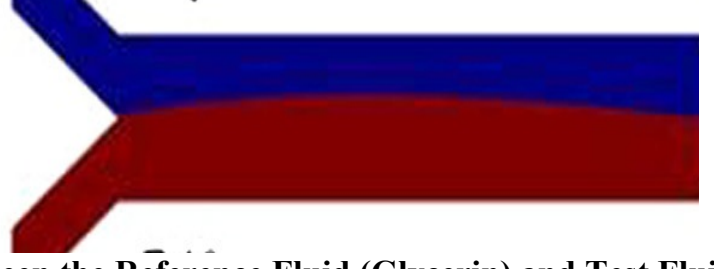

Figure.3: Interface Shift Between the Reference Fluid (Glycerin) and Test Fluid of Varying Viscosity [24]

The dynamic viscosity measurement suggested by Goel et.al. however, just suggests whether the test liquid is adulterated or not [21]. The percentage of adulteration cannot be determined by this system. Also, the syringe pumps are required to insert the test fluid and reference fluid in the channel. This makes the system lab dependent and bulky. The other major issue that does not make the above system suitable as real time solution is the use of reference fluid which should be immiscible with the test fluid. This system though being accurate in determining adulteration is not handy and requires skilled personnel to operate the system. 
The above solution can be modified to a single channel based system with channel comprising of material which reacts to the test fluid adulterant. The devic needs to be fabricated based on the adulterant that has to be determined.

Lujia et. al [1] described that paper-based material has its own advantages over PDMS and other nonporous materials due to porosity. The microfluidic fabricated device is shown in Figure. 4 made from omniphobic paper by embossing technique [29]. This three-dimensional system of micro-channel can work as droplet generator and phase separator. The contact and exchange of fluid in the microchannel with its environment is also enabled due to gas permeability of the paper.

Again this system requires skilled personnel for operation. The determination of immiscible reference fluid poses the problem to determine adulteration at real time. This type of solution needs further modifications to create a robust, highly sensitive low cost solution.

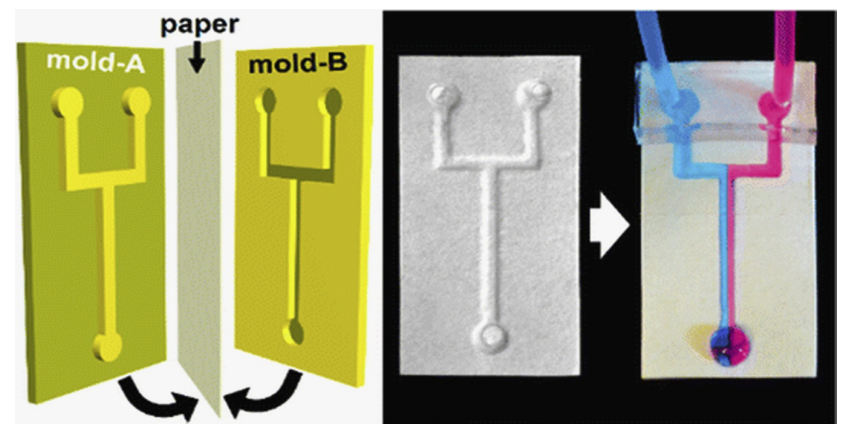

Figure 4: Fabricated Microfluidic Devices using Embossing Technique from Omniphobic Paper [29]

The fabrication process of a microfluidic device is shown in Figure. 5 [30].The device is fabricated by bonding transparent stencil onto a microscope glass slide. The stencil is then removed and replaced by PDMS layer which contains the serpentine channel to the surface of the glass slide, the microfluidics sensor device is fabricated.

The comparison among antibody, MIPs and aptamer in terms of separation sensitivity, specificity, speed, stability, reusability and cost efficiency used as separation elements which can be integrated into microfluidics devices are summarized in Table 1.

\begin{tabular}{llll}
\hline & Antibody & MIPs & Aptamer \\
\hline Separation speed & ++ & +++ & +++ \\
Sensitivity & ++ & + & +++ \\
Specificity & ++++ & ++ & +++ \\
Stability & + & ++++ & +++ \\
Reusability & + & +++ & +++ \\
Cost efficiency & + & +++ & ++ \\
\hline 'Note: '+' stands for the capacity. & &
\end{tabular}

Table 1: Antibody, Molecularly Imprinted Polymers (MIPs) and Aptamer Comparison [1]

The solution suggested by $\mathrm{Hu}, \mathrm{H}$. et. al, is a good solution to the problem of determining adulteration. The flow of the test liquid and pure liquid needs to be determined to form a look up table which can be controlled by a microprocessor to determine percentage of adulteration. However, cleaning of the channel after each use makes the device costly. This device needs further modifications for cost effective solution.

\section{Design Modification and Proposed Design Model}

As reviewed from the above designs it is clear that accurate readings of adulteration can be obtained using microfluidic devices. However, the design needs to be modified so as to obtain instant results. For this we propose a microfluidic device which will measure the capacitance upon changing the fuel which will act as dielectric between two capacitive plates. 


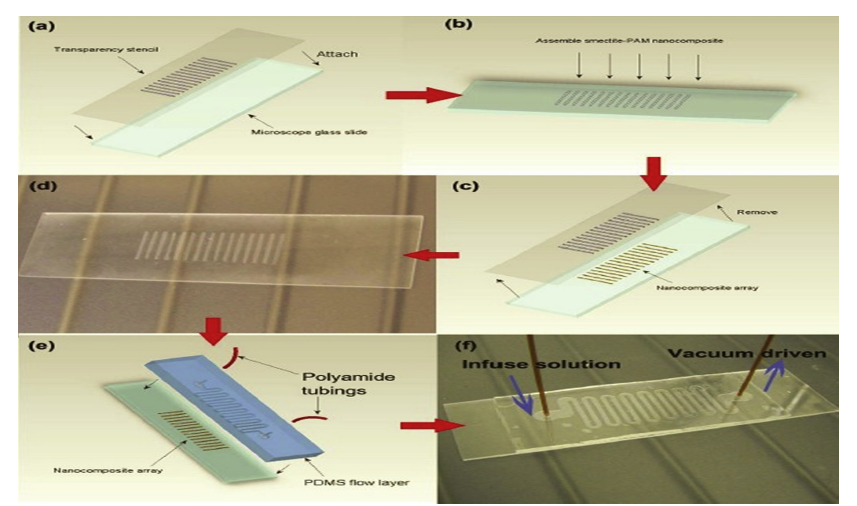

Figure 5: Fabrication Process of a Serpentine Microfluidics Chip [30]

A general idea of such device is shown in Figure 6

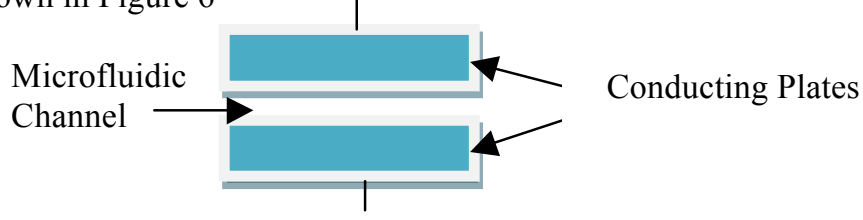

Figure 6: Idea to Design Adulteration Detection for Fuels using Microfluidics

For further analysis, the vibration analysis can be done when the fuel passes through the microfluidic channel. A MEMS based vibration sensor can be used for this purpose which can be mounted in the microfluidic channel itself. Depending on the voltage generated due to vibration of sensor as well as the voltage generated via coplanar capacitance it is possible to obtain accurate results. Kumar et. al has suggested a design of energy harvester which can be utilized as vibration sensor which can be incorporated in this device. Figure. 7 shows the design of this vibration sensor [31].

As suggested in Figure 7 this E-beam cantilever can be designed for three different resonant frequesncies whereby increasing the range in which the operation of vibration takes place.

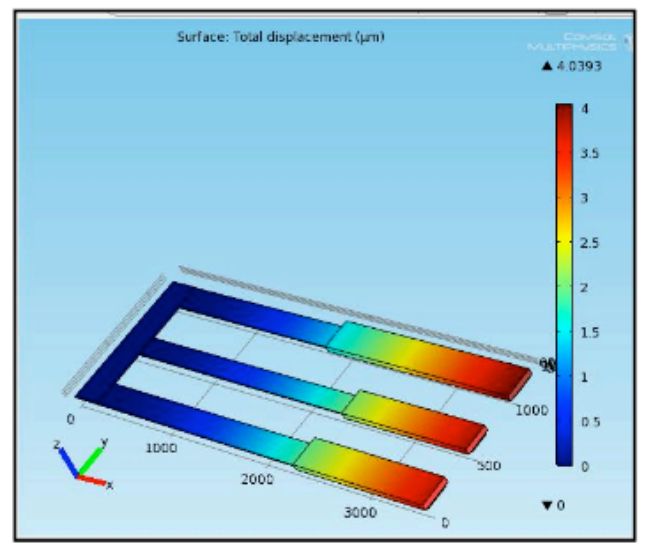

Figure 7: E- Beam Cantilever Beam [31]

\section{Challenges and Future Scope}

Microfluidics being a multidisciplinary field that integrates the study of micro-technology, physics, chemistry, material sciences and engineering. Hence, the amalgamation of these fields to obtain a robust system poses a lot of challenges including testing of such systems. The literature available for microfluidic lab on chip devices is limited and detection of adulteration using these devices are not yet extensively tested. One of the major challenges is the complexity of food matrices. Currently, almost all the microfluidics devices for the detection of adulterants are conducted with off-chip sample pre-treatment. For the development of on-chip sample pretreatment, further efforts are required.

The future scope lies in making micro-viscometer which does not require a reference fluid and detects the flow of the fluid with respect to time and width occupied at unit time. If such viscometer is able to convert this width occupied in some electrical parameter,; then this device can be made generalized for any type of adulterant. Moreover, a processing circuit consisting of a look up table which can determine the type of adulterant needs to be explored. Robustness and sensitivity of the system needs to be tested extensively. Furthermore, the flow will 
cause certain vibrations in the microscopic structure. These vibrations can be detected using MEMS based electromagnetic vibration sensors [31] or piezoelectric MEMS vibration sensors [32]. This will ease out the conversion of flow to electrical parameters.

\section{Conclusion}

This study provides an effective and efficient method of determining adulteration by calculating viscosity of micro-test samples on a microfluidic structured device to carry out real-time adulteration detection and monitoring it in consumable and usable commodities.

According to the width taken by the test sample in the viscometer one can easily calculate its respective viscosity by putting the width value in the standard equation. The device designed is re-usable, can be easily recalibrated and easy to handle. It does not require skilled personnel for its handling. The batch fabrication will also make this device readily available with low cost of production.

\section{References}

[1] Lujia Guo „Jinsong Feng, Zecong Fang, Jie Xu , Xiaonan Lu "Application of microfluidic "lab-on-a-chip” for the detection of mycotoxins in foods"; Trends in Food Science \& Technology, an Elsevier Journal (a)2015

[2] Arevalo, F. J., Granero, A. M., Fernandez, H., Raba, J., \& Zon, M. A. "Citrinin (CIT) determination in rice samples using a micro fluidic electrochemical immunosensor"; Talanta, 83, 966e973 (2011).

[3] Lehotay, S. J., \& Hajslova, J. "Application of gas chromatography in food Analysis"; Trends in Analytical Chemistry, 21, 686e697 (2002)

[4] Centre for Science and Environment (CSE), Delhi report.

[5] Burcik, E.J. 1957. Properties of Petroleum Reservoir Fluids, Chap. 1. New York: John Wiley \& Sons.

[6] McCain, W.D. Jr. "The Properties of Petroleum Fluids"; second edition. Tulsa, Oklahoma 1990: PennWell Publishing Company.

[7] Felix, V.J.; Udaykiran, P.A. \& Ganesan, K. Fuel adulteration detection system. Indian J. Sci. Technol., 2015, 8(S2), 90-95. doi: 10.17485/ijst/2015/v8iS2/59076

[8] Kher, S.; Chaubey, S.; Kishore, J. \& Oak, S.M. Detection of fuel adulteration with high sensitivity using turnaround point long period fiber gratings in b/ge doped fibers. IEEE Sensors J., 2013, 13(11), 4482-4486. doi: 10.1109/JSEN.2013.2270312

[9] Mishra, V.; Tiwari V. \& Patel P.N. Nanoporous silicon microcavity based Fuel Adulteration Sensor, Silicon Journal

[10] Bayraktar, T., \& Pidugu, S. B., "Characterization of liquid flows in microfluidic systems"; International Journal of Heat and Mass Transfer, 49, 815e824 (2006).

[11] Chen, W., Lam, R. H. W., \& Fu, J., "Photolithographic surface micromachining of polydimethylsiloxane (PDMS)"; Lab on a Chip, 12, 391e395 (2012)

[12] Mao, L., \& Koser, H.,"Towards ferrofluidics for m-TAS and lab on-a-chip applications";Nanotechnology, $17,34 \mathrm{e} 47$ (2006).

[13]Zhang, M., \& Wang, Z., "Nanostructured silver nanowires-graphene hybrids for enhanced electrochemical detection of hydrogen peroxide."; Applied Physics Letters, 102, 213104 (2013)

[14] Rasmussen A, Mavriplis C, Zaghloul ME, Mikulchenko O,Mayaram K , "Simulation and optimization of a microfluidic flow sensor"; Sens Actuators A: Phys 2001;88:121-32.

[15] Squires, T. M., Messinger, R. J., \& Manalis, S. R., "Making it stick: convection, reaction and diffusion in surface-based biosensors";Natuer Biotechnology, 26, 417e426 (2008)

[16] Squires, T. M., \& Quake, S. R., "Microfluidics: fluid physics at the nanoliter scale"; Reviews of Modern Physics, 77, 977e1026 (2005).

[17] Xu, J., Vaillant, R., \& Attinger, D., "Use of a porous membrane for gas bubble removal in microfluidic channels: physical mechanisms and design criteria"; Microfluidics and Nanofluidics, 9, $765 \mathrm{e} 772$ (2010)

[18] Xuan J, Leung MKH, Leung DYC, Wang H., "Laminar flow-based fuel cell working under critical conditions: the effect of parasitic current."; Appl Energy 2012;90:87-93.

[19] Abdallah A, Heinisch M, Jakoby B., "Measurement error estimation and quality factor improvement of an electrodynamic-acoustic resonator sensor for viscosity measurement"; Sens Actuators A: Phys 2013;199:318-24.

[20] Naik N, Courcimault C, Hunter H, Berg J, Lee J, Naeli K, et al. “ Microfluidics for generation and characterization of liquid and gaseous micro- and nanojets";Sens Actuators A: Phys 2007;134:119-27.

[21] Martínez M. "Hagen-Poiseuille flow solutions in grad-type equations”; J Stat Phys 2011;142:710-25.

[22] Chang C-C, Yang R-J., “ Electrokinetic mixing in microfluidic systems"; Microfluidics Nanofluidics 2007;3:501-25.

[23] Wang H, Leung DYC, Xuan J., "Modeling of a microfluidic electrochemical cell for CO2 utilization and fuel production"; Appl Energy 2013;102:1057-62. 
[24] Goel Sanket, Venkateswaran P.S., Prajesh Rahul, Agarwal Ajay, "Rapid and automated measurement of biofuel blending using a microfluidic viscometer"; Fuel an Elsevier Journal @2014

[25] Martinez, A. W., Phillips, S. T., Whitesides, G. M., \& Carrilho, E. (2009), "Diagnostics for the developing world: microfluidic paper-based analytical devices"; Analytical Chemistry, 82, 3e10.

[26]Zhou, J., Ellis, A. V., \& Voelcker, N. H. (2010)., "Recent developments in PDMS surface modification for microfluidic devices"; Electrophoresis, 31, 2e16.

[27] Lim, Y. C., Kouzani, A. Z., \& Duan, W. (2010) "Lab-on-a-chip: a component view"; Microsystem Technologies, 16, 1995e2015.

[28] Verpoorte E, de Rooij NF., "Microfluidics meets MEMS"; Proc IEEE 2003;91:930-53.

[29] Thuo, M. M., Martinez, R. V., Lan, W. J., Liu, X., Barber, J., Atkinson, M. B., \& Whitesides, G. M. (2014) "Fabrication of low-cost paper-based microfluidic devices by embossing or cut-and-stack methods" ;Chemistry of Materials, 26, 4230e4237.

[30]Hu, H., Deng, Y., \& Zou, J. (2013), "Microfluidic smectite-polymer nanocomposite" ; strip sensor for aflatoxin detection. IEEE Sensors Journal, 13, 1835e1839

[31] Kumar, Ankita; Balpande, SS; ",Energy Scavenging From Ambient Vibrations Using MEMS Device"; International Journal of Scientific Progress And Research (IJSPR) ISSN: 2349-4689 Volume-05, Number 01,2014

[32] Kumar, Ankita; Balpande, SS; Anjankar, SC; ",Electromagnetic Energy Harvester for Low Frequency Vibrations Using MEMS”; Procedia Computer Science,79,,785-792,2016,Elsevier 